\title{
The Jefferson Lab High Power Light Source
}

\author{
James R. Boyce \\ Jefferson Lab, Newport News, VA 24606 USA
}

\begin{abstract}
Jefferson Lab has designed, built and operated two high average power free-electron lasers (FEL) using superconducting RF (SRF) technology and energy recovery techniques. Between 1999-2001 Jefferson Lab operated the IR Demo FEL. This device produced over $2 \mathrm{~kW}$ in the mid-infrared, in addition to producing world record average powers in the visible $(50 \mathrm{~W})$, ultraviolet $(10 \mathrm{~W})$ and terahertz range $(50 \mathrm{~W})$ for tunable, short-pulse $(<\mathrm{ps})$ light. This FEL was the first high power demonstration of an accelerator configuration that is being exploited for a number of new accelerator-driven light source facilities that are currently under design or construction. The driver accelerator for the IR Demo FEL uses an Energy Recovered Linac (ERL) configuration that improves the energy efficiency and lowers both the capital and operating cost of such devices by recovering most of the power in the spent electron beam after optical power is extracted from the beam. The IR Demo FEL was de-commissioned in late 2001 for an upgraded FEL for extending the IR power to over $10 \mathrm{~kW}$ and the ultraviolet power to over $1 \mathrm{~kW}$. The FEL Upgrade achieved $10 \mathrm{~kW}$ of average power in the mid-IR (6 microns) in July of 2004, and its IR operation currently is being extended down to 1 micron. In addition, we have demonstrated the capability of on/off cycling and recovering over a megawatt of electron beam power without diminishing machine performance. A complementary UV FEL will come on-line within the next year. This paper presents a summary of the FEL characteristics, user community accomplishments with the IR Demo, and planned user experiments.
\end{abstract}

Keywords: FEL, free-electron laser, infrared, UV, THz, high average power, energy recovery linacs.

\section{BACKGROUND}

Jefferson Lab is a national research laboratory operated for the U.S. Department of Energy by the Southeastern Universities Research Association (SURA), a consortium of over 60 universities and colleges in the United States [1]. It uses superconducting radiofrequency (SRF) technology to accelerate electrons to multi-GeV energies for quark/gluon studies in atomic nuclei. In the late 1990s, Jefferson Lab applied the SRF technology towards building a free-electron laser. In 1999, the infrared demonstration free-electron laser (IR DEMO FEL) was commissioned and then operated for the FEL user community until late 2001.

The basic layout of the IR DEMO FEL is shown in Fig. 1. A photocathode gun produced the electron beam which is then accelerated to about $10 \mathrm{MeV}$, injected into the SRF cryomodules containing eight niobium cavities cooled to $2^{\circ} \mathrm{K}$. The cryomodule accelerates the electrons to a tunable energy between $35 \mathrm{MeV}$ and 45 $\mathrm{MeV}$. The electron beam was sent through a wiggler to produce the IR lasing light. Rather than sending the electron beam into a beam dump, the electrons are redirected in a race-track path and re-injected into the cryomodule, but at $180^{\circ}$ out of phase with 
the accelerating RF. The electrons are thus deccelerated back to $9 \mathrm{MeV}$, recovering the energy in the RF, and then are captured in a dump at $9 \mathrm{MeV}$.

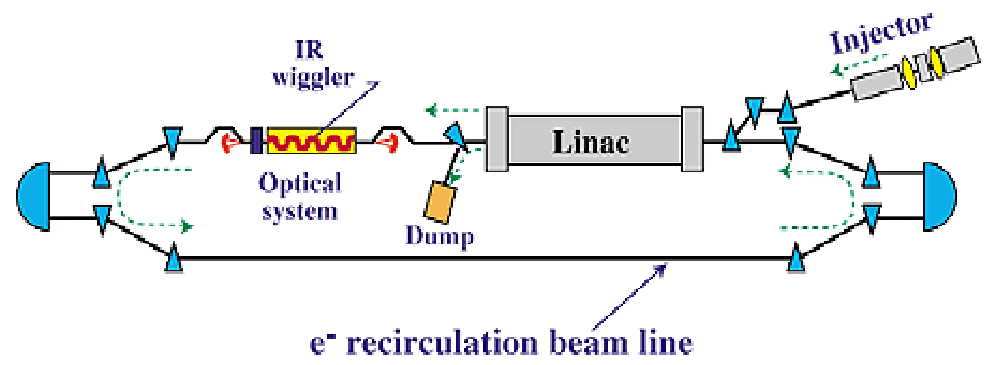

FIGURE 1. Layout of the IR DEMO FEL. Electrons from the injector, traveling counter-clockwise, are accelerated in the LINAC (an SRF cryomodule), pass through the wiggler, produce infrared that is captured in the optical cavity, while the electrons travel around and are re-inserted into the LINAC and are deccelerated, and finally captured in the dump at injector energy.

The IR light produced in the wiggler is captured between two mirrors with a special coating to reflect at desired IR wavelengths. The tunable electron energy allowed us to produce IR between 5 and 10 microns. One mirror was coated for $100 \%$ reflectivity while the other was coated for only $90 \%$ reflectivity. The remaining $10 \%$ was outcoupled and sent, via a vacuum pipe, to 6 user laboratories on the second floor of the FEL building. The amount of useful IR that was provided to users in their upstairs laboratories exceeded $2 \mathrm{~kW}$ [2]. Specifications of the IR DEMO are listed in Table 1.

TABLE 1. Specifications of the IR DEMO FEL (achieved).

\begin{tabular}{lc}
\hline Average Power & 2130 watts \\
Wavelength range & $3-6.2$ microns \\
Micropulse energy & up to 70 microJoules \\
Pulse length & $0.5-1.7 \mathrm{ps}$ \\
PRF & $74.85,37.425,18.7 \mathrm{MHz}$ \\
Bandwidth & $0.3-2 \%$ \\
Amplitude jitter & $<10 \% \mathrm{p}-\mathrm{p}$ \\
Polarization & $>6000: 1$ \\
Transverse mode & $<2 \times$ diffraction limit \\
Beam diameter at lab & $1.5-3.5 \mathrm{~cm}$ \\
\hline
\end{tabular}

Two other features of this FEL were demonstrated: Thomson x-rays and THz or far-infrared radiation. Thomson x-rays are generated by the return infrared bunch scattering off the next electron bunch at the center of the wiggler $[3,4]$. The THz radiation is generated at the exit edge of dipoles. In both cases, the amount of radiation was shown to be substantial. Figure 2 shows the three types of radiation that were obtained simultaneously. 
- 800 fsec pulses at $37.4 \mathrm{MHz}$

- Synchronized to $<<$ psec levels (same beam!)

-All three wavelengths at world class fluxes
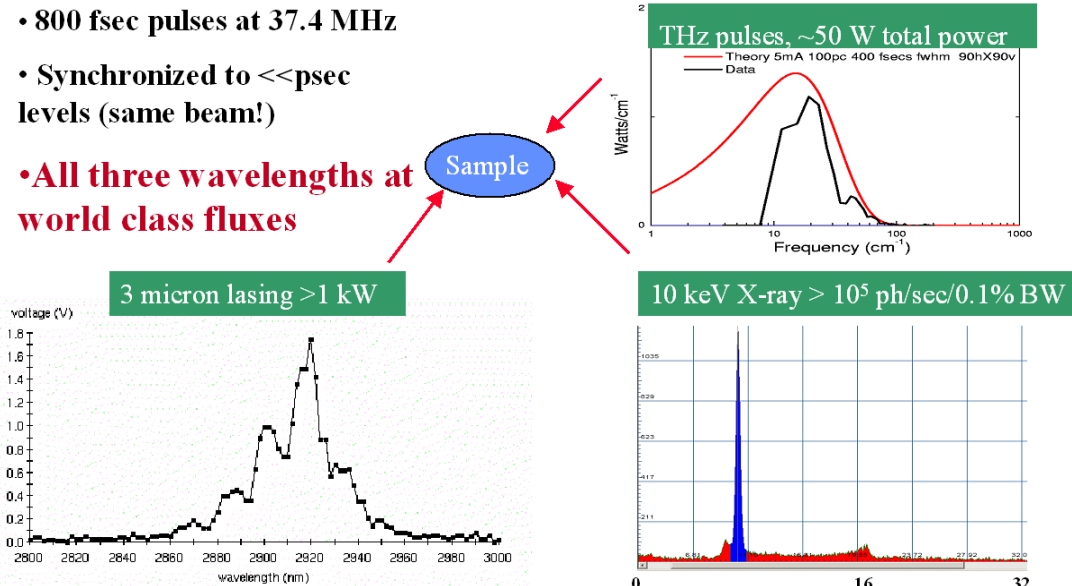

FIGURE 2. Simultaneous production of $3 \mu \mathrm{m}$, broadband $\mathrm{THz}$, and $10 \mathrm{keV}$ x-ray femtosecond pulses.

\section{UPGRADE FEL - DESCRIPTION AND PERFORMANCE}

In November 2001, the IR DEMO FEL was de-commissioned to make room for an upgrade FEL that would increase the IR power output to $10 \mathrm{~kW}$ and to include a $1 \mathrm{~kW}$ UV laser capability. The Upgrade included two additional SRF linac cryomodules and a new wiggler and optical cavity system. Energy recovery was also integral to the design. The layout is shown in Fig. 3. The design specifications for the Upgrade FEL are listed in Table 2.

Mirror cassettes with water-cooled mirrors mounted on translation stages allow remote changing of mirror sets for optimal wavelength tuning. The high reflector (HR) mirror is coated to reflect $100 \%$ of the desired wavelength while the output coupler (OC) mirror is $\sim 90 \%$ reflective and $\sim 10 \%$ transmissive. Since the intra-cavity power is about 10 times that of the out-coupled power, the mirrors are temperature stabilized with a controllable water circuit [5]. 


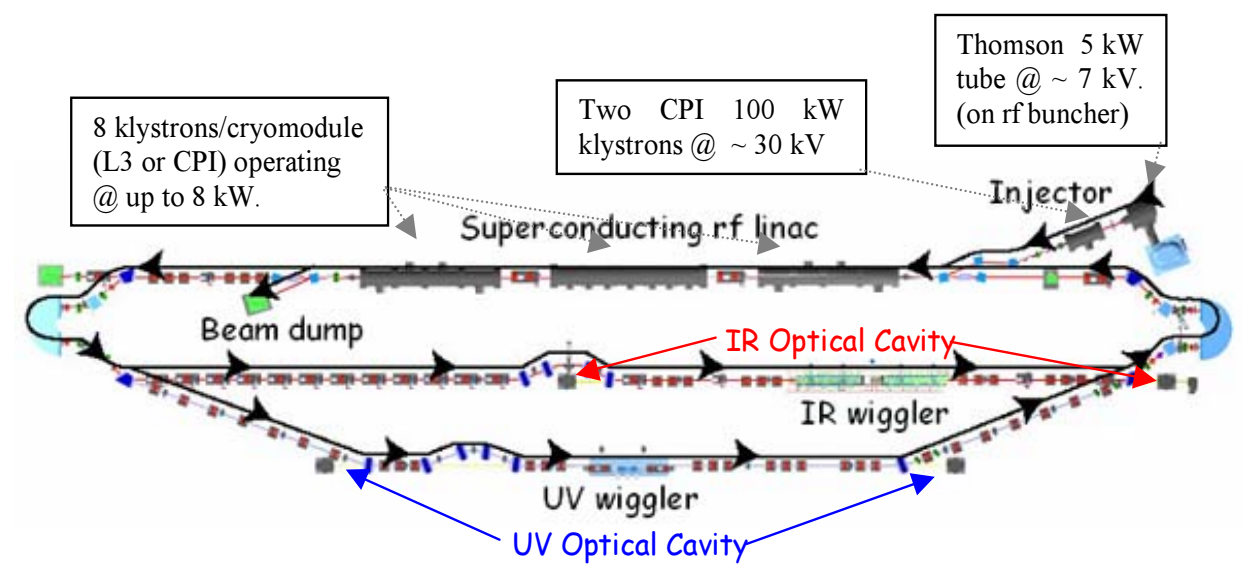

FIGURE 3. Layout of the Upgrade FEL. The arrows indicate the direction of the electron beam. Maximum RF power available is supplied by klystrons as indicated. Actual RF power is less. The IR line has lased at several wavelengths. Installation of the UV line is in late Summer 2005. When complete, the FEL will be operated in either the IR or the UV mode, but not both. Optical transport lines will connect the IR/UV light to user laboratories on the $2^{\text {nd }}$ floor of the FEL facility.

TABLE 2. Design Specifications of the IR/UV Upgrade FEL.

\begin{tabular}{|c|c|c|}
\hline Output Light Parameters & IR Branch & UV Branch \\
\hline Average Power $(\mathrm{kW})$ & $>10$ & $>1$ \\
\hline Wavelength range $(\mu \mathrm{m})$ & $0.7-14$ & $0.25-1$ \\
\hline Micropulse energy $(\mu \mathrm{J})$ & $100-300$ & 25 \\
\hline Pulse length & $0.5-1.7 \mathrm{ps}$ & $0.5-1.7 \mathrm{ps}$ \\
\hline $\mathrm{PRF}$ ("CW" operation, $\mathrm{MHz}$ ) & $4.7-75$ & $4.7-75$ \\
\hline Electron Beam Parameters & IR-Branch & UV Branch \\
\hline Energy $(\mathrm{MeV})$ & $80-200$ & 200 \\
\hline Charge per bunch $(\mathrm{pC})$ & 135 & 135 \\
\hline Average current (mA) & 10 & 5 \\
\hline Peak Current (A) & 270 & 270 \\
\hline Beam Power (kW) & 2000 & 1000 \\
\hline Energy Spread (\%) & 0.5 & 0.13 \\
\hline Normalized emittance (mm-mrad) & $<30$ & $<11$ \\
\hline Induced energy spread (full) & $10 \%$ & $5 \%$ \\
\hline \multicolumn{3}{|c|}{ Broadband THz Beamline Parameters } \\
\hline Wavelength Range (THz) & \multicolumn{2}{|c|}{$0.1-10$} \\
\hline Pulse Length (ps) & \multicolumn{2}{|c|}{$0.2-2$} \\
\hline Power/pulse ( $\mu$ Joules) & \multicolumn{2}{|c|}{2} \\
\hline Repetition Rate (Hz) & \multicolumn{2}{|c|}{$1-75,000,000$} \\
\hline Total Power (watts) & \multicolumn{2}{|c|}{150} \\
\hline
\end{tabular}


The FEL has achieved $10 \mathrm{~kW}$ average output power as shown in Fig. 4. These data were taken over several minutes in a "one second on, three seconds off" mode. The reason for this unconventional mode was that the HR mirror was heating up and distorting beyond its design capabilities due to excessive $\mathrm{THz}$ radiation. However, all the RF and electronics operated smoothly as we slammed on, then off, and energy recovered $1 \mathrm{MW}$ of electron beam power. This speaks highly of the ruggedness of the FEL. Beam line changes were then incorporated to reduce the $\mathrm{THz}$ radiation directed onto the OC mirrors. Once these changes are fully commissioned, we expect minutes to hours of $\mathrm{CW} 10 \mathrm{~kW}$ operations for our user community.

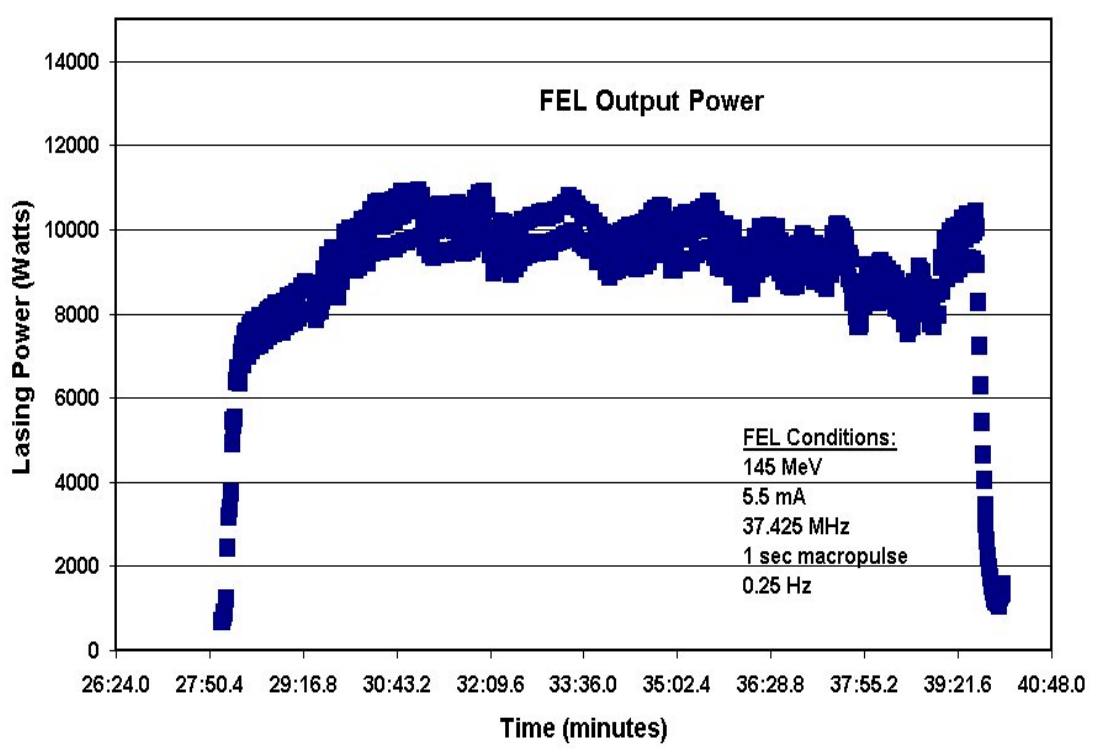

FIGURE 4. FEL operations at $10 \mathrm{~kW}$.

\section{FEL UPGRADE - THE PATH FORWARD}

Now that the $10 \mathrm{~kW}$ goal has been achieved, the program focus will concentrate on key areas for improving and extending machine performance. Performance of the injector can be improved by reducing the halo produced by scattered drive laser light, and improving gradient of the quarter cryomodule either by reconditioning or replacement. The acceleration energy and current capacity of the three cryomodules can be further enhanced by RF conditioning or replacement. Beam Break Up (BBU), whereby higher order modes of the SRF cause instabilities in the electron beam, has long been predicted and only recently been seen (See Fig. 5). Further systematic studies and theory/model/experiment comparisons can be accomplished with the upgrade FEL for optimizing a means to mitigate/eliminate this phenomenon [6]. Table 3 summarizes the BBU issues and several approaches for mitigation have already been demonstrated. Finally, the optics of the optical cavity can be improved both in thermal and physical stability of the mounting. 




FIGURE 5. Onset of BBU as shown in HOM power in one of the cavities of a cryomodule, measured by Schottky diodes, versus time for $\mathrm{I}_{\mathrm{b}}=3.6,4.2$, and $5 \mathrm{~mA}$. The risetime depends on the beam current as $\tau=\tau_{\mathrm{o}}\left[\mathrm{I}_{\mathrm{th}} /\left(\mathrm{I}-\mathrm{I}_{\mathrm{th}}\right)\right]$, where $\mathrm{I}_{\mathrm{th}}$ is the threshold for the HOM.

TABLE 3. BBU Suppression Techniques.

\begin{tabular}{|c|c|c|}
\hline Suppression Type & $\begin{array}{c}\text { Effect on } 2106 \\
\text { MHz HOM }\end{array}$ & $\begin{array}{c}\text { Implementation } \\
\text { Considerations } \\
\end{array}$ \\
\hline Q-Damping: Damping Circuit & $1.5 \times \mathrm{I}_{\text {th }}$ & $\begin{array}{l}\text { - } \quad \text { Works only on } 1 \text { mode } \\
\text { per cavity } \\
\text { - Not as effective at raising } \\
\mathrm{I}_{\text {th }} \text { as beam optical } \\
\text { methods } \\
\text { - } \quad \begin{array}{l}\text { Does not effect beam } \\
\text { optics }\end{array} \\
\end{array}$ \\
\hline Beam Optics: Pseudo-reflector [7] & Stabilized & $\begin{array}{l}\text { - Can stabilize the mode } \\
\text { against BBU } \\
\text { What are the effects on } \\
\text { other HOMs? } \\
\text { Do they prevent reaching } \\
\text { the requirements needed } \\
\text { for a suitable lasing } \\
\text { configuration? }\end{array}$ \\
\hline
\end{tabular}




\section{FEL UPGRADE - A FEW APPLICATIONS}

Applications of the FEL Upgrade's light can be grouped into three basic categories: Biomedical, Industrial, and Military. Only a few examples will be discussed here. A more complete listing of applications can be found by mining the JLab FEL web sites (see www.jlab.org/FEL).

Biomedical applications: The applications range from studies of vibration states in organic molecules such as proteins and myoglobin [8], to preferential fat cell destruction by infrared wavelengths for which fatty tissue is more absorptive than other normal tissue, to tattoo removal by a similar technique whereby the color dyes in tattoos are broken up by the infrared FEL light so the body can absorb and eliminate without healthy tissue damage.

Industrial applications: 1) A joint research effort between JLab, NASA, the College of William and Mary, and Penn State University, has developed an FEL-based production technique for generating single-wall carbon nanotubes at rates exceeding grams/hour [9]. 2) Non-thermal surface conditioning whereby grain boundaries are eliminated by rapid melting/solidification with the IR light, thus reducing corrosion that occurs first at grain boundaries. 3) Studies of hydrogen impurities in silicon is an active program [10].

Military applications: 1) The U.S. Navy is interested in developing the FEL into a defensive device and proposes studies of atmospheric transmission of various IR wavelengths. 2) The U.S. Army is interested in exploring THz imaging for land mine detection. 3) The U.S. Air Force, in collaboration with JLab and the Aerospace Corporation is setting up for micro-machining for nano-satellite fabrication.

\section{SUMMARY}

The Jefferson Lab High Power FEL Light Source has been described including the initial history, concept, energy recovery, and light production. Challenges and applications have also been briefly described. Many "opportunities" exist for further work to address unresolved issues. Much progress has been made to advance the technology and understanding to levels which permit the next generation of light sources. We are addressing key issues for ERLs, including generating high average brightness beams and then preserving and controlling them through the acceleration and recovery process. The JLab IR/UV/THz/X-ray Upgrade ERL is becoming a productive user facility with exceptional performance in several wavelength bands.

\section{ACKNOWLEDGEMENTS}

The author gratefully acknowledges the help of the entire FEL Team without which this paper, let alone the accomplishments herein described, would not have occurred. This entire effort was sponsored and supported by the Office of Naval Research, the Air Force Research Laboratory, the U.S. Army Night Vision Laboratory, the Commonwealth of Virginia, and the Laser Processing Consortium under a U.S. Department of Energy Office of Science Contract. 


\section{REFERENCES}

1. Available online: www.sura.com.

2. M.D. Shinn, "High Average Power Free-Electron Lasers - A New Laser Source for Materials Processing" SPIE 4065, pp. 434-440 (2000).

3. J.R. Boyce, "Intra-cavity Thomson Scattering," Section 2.5.2, in Femtosecond Beam Science, edited by M. Uesaka, World Scientific, in press.

4. J.R. Boyce, D.R. Douglas, H. Toyokawa, W.J. Brown, F. Hartemann, "Sub-Picosecond, High Flux, Thomson X-ray Sources at Jefferson Lab's High Power FEL," Proc. 2003 Particle Accelerator Conf., Portland, OR, May 12-16, 2003, p. 938.

5. M.D. Shinn, et al., "Design of the Jefferson Lab IR Upgrade FEL Optical Cavity," Nuclear Instruments and Methods A507, 196 (2003).

6. C.D. Tennant, K.B. Beard, D.R. Douglas, K.C. Jordan, L. Merminga, E.G. Pozdeyev, and T.I. Smith, "First observations and suppression of multipass, multibunch beam breakup in the Jefferson Laboratory free electron laser upgrade," available online http://link.aps.org/abstract/PRSTAB/v8/e074403.

7. R. Rand and T. Smith., "Beam Optical Control of Beam Breakup in a Recirculating Electron Accelerator," Particle Accelerators, Vol. II, pp. 1-13 (1980).

8. R.H. Austin, A.H. Xie, L. van der Meer, M. Shinn, and G. Neil, "Self-trapped states in proteins" Nuclear Instruments and Methods A507, 561 (2003).

9. P. C. Eklund, B. K. Pradhan, U. J. Kim, Q. Xiong, J. E. Fischer, A. D. Friedman, B. C. Holloway, K. Jordan and M. W. Smith "Large-Scale Production of Single-Walled Carbon Nanotubes Using Ultrafast Pulses from a Free Electron Laser", Nano Letters 2, 561 (2002).

10. G. Lüpke, X. Zhang, B. Sun, A. Frazer, N.H. Tolk, and L.C. Feldman, "Structure-Dependent Vibrational Lifetimes of Hydrogen in Silicon," Phys. Rev. Lett. 88, 135501 (2002). 